\title{
Implications Of Occupational And Geographic Mobility Among College-Trained Workers For Southern Manpower Supply- Demand Balances\#
}

\author{
RICHARd A. ENGELS*
}

National studies of manpower supply and demand can exclude consideration of migration streams without grave consequences for their findings. The balances of immigration and emigration may be significant for a few selected occupations, but in general the impact is trivial relative to the labor force as a whole and to other sources of trained workers.

Movement between occupations is recognized as a more serious concern to be dealt with regardless of the area covered by the research (U.S. Bureau of Labor Statistics, 1974). The burgeoning interest and activity in regional and state-level manpower studies call attention to both geographic and occupational movements of trained manpower. The intent of this paper is to evaluate the degree to which these two factors modify the manpower supply-demand picture for the Southern region, and to gauge the potential threat to the employment outlook of new Southern college graduates during the 1970's.

Although recent evidence indicates that the earlier U-shaped relationship of migration and occupational status may have shifted to a more nearly continuous linear function (Long and Boertlein, 1975), the fact remains that the high rates of migration for professional workers during the past thirty years continue. For some professional occupations at a regional or state level, it is easily conceivable that migration is a significant force in restructuring the stock of college and university-trained human capital. The report of an Oregon study recognizes the potential of migration to disrupt the straightforward comparison of supply and demand in college-level manpower analysis, but it was felt that adequate data were lacking for adjustment (Siler, n.d.). A dynamic labor supplydemand model for Colorado was able to be modified to account for the interstate migration disturbance to worker supplies (Rake, n.d.). Resident labor force entrants were derived independently and were combined with an assumed volume of inmigration to determine the total worker supply. Occupational detail is not available from the study to obtain a thorough assessment of impact, however.

\footnotetext{
\#Prepared for presentation at the Southern Regional Science Association Annual Meeting, Richmond, Virginia, April 1976. Data and analysis partially completed while author was Manpower Project Officer, Southern Regional Education Board, Atlanta, Georgia.

$*$ U.S. Bureau of the Census.
} 
An Alabama project concerned itself at length with a suspected influence from both occupational and geographic mobility, but concluded that the lack of required detail for both variables precluded their use at the time the research was conducted (Alabama Commission on Higher Education, 1972). A later and more complex modeling project for Georgia college-level workers during the 1970's, however, deals with "retained manpower" moved forward from the base date and incorporates adjustments for both occupational shifts and geographic movement of trained manpower (White and Drummond, n.d.).

For perhaps the first time on a comprehensive scale, mobility data from the 1970 Census of Population afford a detailed look at both types of movement and at levels of geography below the Nation as a whole. The possible magnitude of the adjustments and their effects upon the relationship of supply and demand for college-level workers are documented in a recent preliminary pilot analysis for Georgia (Engels, 1975). Background data for the test refer to the 1965-70 period and were taken from special tabulations of the Public Use Sample (PUS) file of 1970 Census returns (U.S. Bureau of the Census, 1972). The analysis covers only bachelor's level degrees and indicates that migration alone, were it to continue at its 1965-70 level, would satisfy 18 percent of the demand for bachelor's level workers. The impact of both mobility factors is felt more strongly in individual occupations and can be illustrated more clearly at that level of detail. In a typical year during the $1965-70$ period, Georgia experienced a net gain of approximately 440 accountants, 80 architects, and 40 civil engineers through the combined influences of in and out migration and in and out occupational shifts by workers. At the projected 1975 levels of educational attainment, and if the migration of the 1965-70 period can be assumed to continue into the 1970's, this would mean that approximately 35 percent of the projected demand for accountants in Georgia would be satisfied by net worker migration and occupational movement. Similarly, the demand for architects would be totally consumed by migration and occupational shifts, and the demand for civil engineers would be 20 percent fulfilled. These kinds of supply supplements relative to demand imply a considerable amount of competition for new local college graduates seeking positions in those occupations, and also represent a substantial constraint and/or resource element to be contended with in higher education planning at the regional and state levels.

A regional rather than a state perspective is desirable from two standpoints, however. The first is a concern for maximum confidence in the basic data series. Table 1 contains comparative occupational and geographic mobility figures from tabulations of the PUS file for illustrative professional occupations in Georgia and in the 14 state region. ${ }^{1}$ Even though the total number of inmigrants for Georgia is 150,700 workers

1Alabama, Arkansas, Florida, Georgia, Kentuc'ry, Louisiana, Mississippi, Missouri, North Carolina, South Carolina, Tennessee, Texas, Virgini 1 , and West Virginia. 
(total regardless of educational background), there are several entries of 100-500 persons. Since the sample rate used in constructing the basic PUS file used here is one percent, many of the figures for individual Georgia occupations are based on only one to five persons being caught in the sampling process. Although the structure or occupational profile obtained appears reasonable and may be used without extreme risk, applications involving findings for individual occupations are hazardous. The corresponding data for the region are not as subject to this criticism and may be utilized without many of the small-number caveats that must be attached to the state findings.

Second, the phenomenal turn-around in net migration during the 197075 period is not necessarily shared proportionately by all of the states. In an effort to minimize the influence of local factors on the changing volume and character of migrants, it is advisable to examine the states in the aggregate for any clues that the $1965-70$ period might be able to provide for the 1970's.

\section{TABLE 1}

Illustrative Comparative Tabulations of Inmigrants and In-Transfers from the Public Use Sample File (Expanded from sample data) for Georgia and the 14-State Southern Region: 1965-70

\begin{tabular}{|c|c|c|c|c|}
\hline \multirow{2}{*}{$\begin{array}{c}1970 \\
\text { Occupation }\end{array}$} & \multicolumn{2}{|c|}{ Inmigrants } & \multicolumn{2}{|c|}{$\begin{array}{l}\text { Transfers into } \\
\text { Occupations }\end{array}$} \\
\hline & Georgia & Region & Georgia & Region \\
\hline Accountants & 3,000 & 13,300 & 2,900 & 40,300 \\
\hline Architects & 300 & 1,200 & 100 & 2,300 \\
\hline Computer Programmers & 400 & 6,200 & 1,100 & 13,900 \\
\hline Systems Analysts & 400 & 2,900 & 400 & 6,600 \\
\hline Civil Engineers & 300 & 3,900 & 800 & 11,300 \\
\hline Industrial Engineers & 1,300 & 5,300 & 900 & 14,600 \\
\hline Lawyers & 300 & 4,500 & 700 & 7,500 \\
\hline Pharmacists & 100 & 700 & 300 & 2,200 \\
\hline Physicians & 1,200 & 8,200 & 100 & 4,900 \\
\hline Registered Nurses & 2,800 & 19,000 & 1,200 & 20,000 \\
\hline Clergymen & 1,400 & 7,200 & 1,300 & 12,300 \\
\hline Economists & 100 & 2,700 & 500 & 4,300 \\
\hline Social Workers & 700 & 3,100 & 900 & 13,300 \\
\hline Elementary Teachers & 4,600 & 30,600 & 1,700 & 37,100 \\
\hline Airline Pilots & 1,200 & 2,200 & 0 & 3,800 \\
\hline Editors and Reporters & 600 & 4,100 & 300 & 5,700 \\
\hline Photographers & 200 & 1,100 & 300 & 2,900 \\
\hline Bank Managers & 1,300 & 5,400 & 1,700 & 21,400 \\
\hline College Administrators & 300 & 800 & 300 & 5,100 \\
\hline Stock and Bond Salesmen & 200 & 1,800 & 700 & 6,100 \\
\hline Bank Tellers & 400 & 3,900 & 900 & 12,300 \\
\hline Bookkeepers & 2,300 & 18,100 & 9,900 & 115,600 \\
\hline Computer Operators & 300 & 2,300 & 0 & 9,200 \\
\hline Secretaries & 7,500 & 47,500 & 10,300 & 123,600 \\
\hline
\end{tabular}




\section{Definitions and Methods}

The intent of this investigation is to present the 1965-70 findings on migration and occupational shifts for the region and to assess their general importance as worker supply variables by contrasting the net effect of in and out movement in both factors with the demand for college educated workers in selected occupations during the 1970-80 decade.

\section{Migration}

The basic data source for both nonmigrant transfers and inter-regional labor mobility was the 1/100 PUS files for states from the 1970 Census of Population. A special subfile was created selecting all employed nonmilitary 1965 residents of the region who lived outside the region in 1970 (outmigrants), and 1970 residents of the region living in another region in 1965 (inmigrants). Tabulations were prepared identifying the 1965 occupation of outmigrants, which may be viewed as additional demand; i.e., potential positions in occupations left vacant by the outmigrant. Inmigrants were tabulated by their 1970 occupations and may be considered as part of the occupational supply due to migration to the region, or due to simultaneous migration and occupational change. These tabulations are illustrated by the results for selected occupations shown in Table 1. The network of destinations for outmigrants and the geographic origins of inmigrants were unimportant to the present analysis and were not retained.

To conform to the definition and usage of the occupational demand concept, it was necessary to express the PUS results in average annual terms. The conversion from raw PUS tabulations was accomplished by expanding the tabulation results to "full count" levels from the sample findings. This represents an estimated 1965-70 value, which was simply divided by five to approximate an average annual level for the period.

\section{Occupational Movement}

Similar files were constructed and treatment given to employed nonmilitary nonmigrant residents of the region who had changed occupations between 1965 and 1970. Positions left by those moving out of a 1965 occupation may be considered part of the demand for that occupation, and workers shifting into a 1970 occupation are defined as additional supply for that occupation.

Both in the case of occupational change and for geographic migration, independent tabulations were developed for the region rather than aggregating results for the composite 14 states. This procedure recognizes that interstate migrants should not necessarily be identified to appear in the same classification at the regional level. For instance, an accountant moving from Georgia to the same occupation in Florida would not be included in the regional tables at all, yet he would appear as an entry 
in both the Georgia and Florida migration tables if states were the unit of analysis. Moreover, if he were to move from an accounting position in Georgia to become a business manager in Florida he would be tabulated as an occupational transfer for the region but he would not be included in the regional migration figures since he had not entered or departed from the region.

\section{Occupational Demand}

Openings in occupations include demand resulting from expansion of employment as industries grow and from retirement, death, and other separations from the labor force. The resulting demand represents average annual opportunities for the 1970-80 period.

The procedures followed in projecting the demand for graduates began with the steps and data used in the Bureau of Labor Statistics (BLS) state cooperative program for manpower projections (U.S. Department of Labor, n.d.). The method moves occupational levels forward from 1970 to the projected date by accounting for industrial growth, disaggregating total industrial employment levels through shifting occupational shares, and providing for worker attrition through occupationspecific separation rates. The only significant departures from the BLS procedures were the elimination of the occupation not reported category and the use of employment growth rates by industry from the National Planning Association rather than from individual state analysts (Lee and Hong, 1973).

\section{Proportion of Demand to be Filled by College Graduates}

All openings projected as demand for each occupation are not treated as opportunities for college graduates. Instead educational attainment levels were projected asymptotically to 1980 for each occupation from the 1960-70 experience. This provides for continuation of the long-term trend of change but not necessarily by the same amount. Table 2 presents the projected 1980 levels and their percentage change from levels observed in 1970 for selected professional occupations.

From the projected 1980 levels, it was then possible to solve the demand equation taking into account both (1) the changing levels of educational attainment, and (2) the correspondingly altered proportion of new workers who must be college educated relative to all workers in the occupation in order to reach the projected 1980 levels. Educational attainment levels of migrants and occupational transfers were not tabulated directly from the PUS files of census responses. Attainment levels for 1975 were imputed to both migrants and occupational transfers as a part of the 1970 to 1980 projection process.

This differential in date of reference may appear inappropriate since the base mobility data refer to the $1965-70$ period. The purpose of the analysis, however, is to obtain a preliminary evaluation of the magnitude 
TABLE 2

Projected Proportion of Workers in Occupations who are College Trained by Number of College Years Completed

Projected 1980 Proportions and Percentage Change 1970-80 Selected Occupations for the United States

\begin{tabular}{|c|c|c|c|c|}
\hline \multirow[b]{2}{*}{ Occupation } & \multicolumn{2}{|c|}{ Projected 1980} & \multicolumn{2}{|c|}{$\begin{array}{l}\text { Percentage } \\
\text { Change from }\end{array}$} \\
\hline & 4 & $5+$ & 4 & $5+$ \\
\hline Accountants & .360 & .107 & 7.9 & 12.3 \\
\hline Architects & .178 & .581 & -18.6 & 10.7 \\
\hline Computer Programmers & .327 & .146 & 11.3 & 18.1 \\
\hline Systems Analysts & .345 & .227 & 11.0 & 16.6 \\
\hline Civil Engineers & .383 & .289 & 0.3 & 30.9 \\
\hline Industrial Engineers & .309 & .207 & 1.0 & 23.5 \\
\hline Lawyers & .039 & .878 & -42.1 & 1.6 \\
\hline Pharmacists & .373 & .532 & -8.7 & 59.8 \\
\hline Physicians & .020 & .926 & -42.2 & 0.1 \\
\hline Registered Nurses & 137 & .048 & 20.7 & 3.9 \\
\hline Clergymen & 128 & .602 & -2.1 & 1.9 \\
\hline Economists & .361 & .388 & 7.6 & -0.8 \\
\hline Social Workers & 441 & .265 & 15.8 & -11.8 \\
\hline Elementary Teachers & .594 & .344 & 8.4 & 16.0 \\
\hline Airline Pilots & .314 & .086 & 46.6 & 38.6 \\
\hline Editors and Reporters & .378 & .194 & 6.1 & 14.4 \\
\hline Photographers & .093 & .023 & 11.7 & -22.7 \\
\hline Bank Managers & .287 & 167 & 18.8 & 45.2 \\
\hline Stock and Bond Salesmen & 431 & .290 & 20.6 & 66.8 \\
\hline College Administrators & .230 & .498 & 45.0 & -17.8 \\
\hline Bank Tellers & .022 & .003 & -19.0 & -35.9 \\
\hline Bookkeepers & .057 & .017 & 33.6 & 58.3 \\
\hline Computer Operators & .037 & .022 & 5.6 & 52.3 \\
\hline Secretaries & .039 & .007 & -12.9 & -14.9 \\
\hline
\end{tabular}

of additional net worker supply provided by geographic and occupational mobility should the experience of the late 1960's extend into the 1970's. Reduced to its simplest form, the analysis seeks a preliminary answer to the question of whether or not these two types of mobility are worth worrying about as supply sources for college educated workers in projections of higher education manpower supply-demand balances.

\section{Net Supply Adjustments Relative to Demand}

The net shifts in and out of detailed occupations shown in Table 3 and the effects of net migration to and from the region result in an addition of approximately 61,300 annually to the supply of college educated workers (bachelor's degree or higher). Occupations with particularly large supply adjustments resulting from the two forms of mobility are accountants, computer programmers, electrical engineers, lawyers, personnel workers, physicians, social workers, elementary and secondary teachers, vocational counselors, and general managers and administrators. 
TABLE 3

Estimated Average Annual Migration and Occupational Mobility

for Workers with College Degrees, Selected Occupations for the Southern Region 1965-1970*

\begin{tabular}{|c|c|c|c|c|c|c|c|}
\hline \multirow[b]{2}{*}{ Occupation } & \multirow{2}{*}{$\begin{array}{c}\text { Total Net } \\
\text { Adjustment }\end{array}$} & \multicolumn{2}{|c|}{ Migration } & \multicolumn{2}{|c|}{$\begin{array}{l}\text { Occupational } \\
\text { Change }\end{array}$} & \multirow{2}{*}{$\begin{array}{l}\text { Unadjusted } \\
\text { Demand }\end{array}$} & \multirow{2}{*}{$\begin{array}{l}\text { Percent of } \\
\text { Unadjusted } \\
\text { Demand } \\
\text { Provided } \\
\text { by Net } \\
\text { Adjustment }\end{array}$} \\
\hline & & In & Out & In & Out & & \\
\hline Total & 61,327 & 42,392 & 17,647 & 145,840 & 109,257 & 294,230 & 20.8 \\
\hline Professional, technical & 34,623 & 30,065 & 11,944 & 65,926 & 49,425 & 151,778 & 22.8 \\
\hline Accountants & 1,836 & 1,155 & 457 & 3,386 & 2,248 & 6,696 & 27.4 \\
\hline Architects & 270 & 180 & 90 & 361 & 180 & 945 & 28.6 \\
\hline Computer Programmers & 1,193 & 543 & 98 & 1,122 & 374 & 1,579 & 75.6 \\
\hline Computer Systems Analysts & 613 & 323 & 183 & 667 & 194 & 1,480 & 41.4 \\
\hline $\begin{array}{l}\text { Computer Specialists, Other } \\
\text { Aeronautical \& }\end{array}$ & 10 & 40 & 60 & 100 & 70 & 133 & 7.5 \\
\hline Astronautical Engineers & 92 & 251 & 264 & 528 & 423 & 544 & 16.9 \\
\hline Chemical Engineers & 141 & 474 & 281 & 439 & 492 & 409 & 34.5 \\
\hline Civil Engineers & 790 & 510 & 153 & 1,351 & 918 & 1,925 & 41.0 \\
\hline \multicolumn{8}{|l|}{ Electrical and } \\
\hline Electronic Engineers & 1,736 & 1,236 & 462 & 1,936 & 974 & 2,815 & 61.7 \\
\hline Industrial Engineers & 970 & 515 & 168 & 1,326 & 702 & 1,691 & 57.4 \\
\hline Mechanical Engineers & 859 & 472 & 254 & 1,162 & 520 & 1,191 & 72.1 \\
\hline \multicolumn{8}{|l|}{ Metallurgical and } \\
\hline Materials Engineers & 72 & 14 & 14 & 143 & 72 & 127 & 56.7 \\
\hline Mining Engineers & 0 & 12 & 0 & 62 & 75 & 16 & 0.0 \\
\hline Petroleum Engineers & -62 & 94 & 47 & 187 & 296 & 132 & - \\
\hline Sales Engineers & 305 & 200 & 74 & 389 & 210 & 296 & 103.0 \\
\hline Other Engineers & 389 & 698 & 378 & 1,511 & 1,442 & 2,211 & 17.6 \\
\hline
\end{tabular}

*Bachelor's degree or higher

Note: Figures in the body of the table may not add precisely to the total net adjustment due to computer rounding of numbers. 
TABLE 3-Continued

Estimated Average Annual Migration and Occupational Mobility

for Workers with College Degrees, Selected Occupations

for the Southern Region 1965-1970

\begin{tabular}{|c|c|c|c|c|c|c|c|}
\hline \multirow[b]{2}{*}{ Occupation } & \multirow{2}{*}{$\begin{array}{c}\text { Total Net } \\
\text { Adjustment }\end{array}$} & \multicolumn{2}{|c|}{ Migration } & \multicolumn{2}{|c|}{$\begin{array}{l}\text { Occupational } \\
\text { Change }\end{array}$} & \multirow{2}{*}{$\begin{array}{l}\text { Unadjusted } \\
\text { Demand }\end{array}$} & \multirow{2}{*}{$\begin{array}{c}\text { Percent of } \\
\text { Unadjusted } \\
\text { Demand } \\
\text { Provided } \\
\text { by Net } \\
\text { Adjustment }\end{array}$} \\
\hline & & In & Out & In & Out & & \\
\hline Farm Management Advisors & 37 & 19 & 0 & 93 & 74 & 93 & 39.8 \\
\hline Foresters and Conservationists & 108 & 66 & 41 & 373 & 290 & 736 & 14.7 \\
\hline Home Management Advisors & 109 & 31 & 0 & 140 & 62 & 202 & 54.0 \\
\hline Judges & 221 & 0 & 0 & 309 & 88 & 216 & 102.3 \\
\hline Lawyers & 1,220 & 850 & 203 & 1,349 & 776 & 4,766 & 25.6 \\
\hline Librarians & 604 & 255 & 107 & 913 & 457 & 2,637 & 22.9 \\
\hline Archivists and Curators & 63 & 63 & 10 & 21 & 10 & 93 & 67.7 \\
\hline Actuaries & -98 & 0 & 33 & 0 & 65 & 54 & - \\
\hline Mathematicians & 0 & 70 & 52 & 87 & 105 & 260 & 0.0 \\
\hline Statisticians & 114 & 103 & 23 & 103 & 69 & 778 & 14.7 \\
\hline Agricultural Scientists & 134 & 34 & 11 & 157 & 45 & 134 & 100.0 \\
\hline Atmospheric and Space Scientists & -11 & 23 & 34 & 23 & 23 & 106 & - \\
\hline Biological Scientists & 312 & 197 & 33 & 230 & 82 & 660 & 47.3 \\
\hline Chemists & 503 & 384 & 148 & 872 & 606 & 1,172 & 42.9 \\
\hline Geologists & -72 & 144 & 198 & 162 & 180 & 346 & - \\
\hline Marine Scientists & 76 & 30 & 0 & 76 & 30 & 106 & 71.7 \\
\hline Physicists and Astronomers & 342 & 270 & 0 & 162 & 90 & 266 & 128.6 \\
\hline Other Life \& Physical Scientists & 18 & 36 & 0 & 18 & 36 & 57 & 31.6 \\
\hline Operations and Systems Researchers & 292 & 186 & 81 & 422 & 235 & 1,210 & 24.1 \\
\hline \multicolumn{8}{|l|}{ Personnel and } \\
\hline Labor Relations Workers & 2,268 & 519 & 168 & 2,406 & 489 & 3,023 & 75.0 \\
\hline Chiropractors & -35 & 35 & 35 & 35 & 69 & 254 & - \\
\hline
\end{tabular}

*Bachelor's degree or higher

Note: Figures in the body of the table may not add precisely to the total net adjustment due to computer rounding of numbers. 
TABLE 3-Continued

Estimated Average Annual Migration and Occupational Mobility for Workers with College Degrees, Selected Occupations for the Southern Region 1965-1970*

\begin{tabular}{|c|c|c|c|c|c|c|c|}
\hline \multirow[b]{2}{*}{ Occupation } & \multirow{2}{*}{$\begin{array}{c}\text { Total Net } \\
\text { Adjustment }\end{array}$} & \multicolumn{2}{|c|}{ Migration } & \multicolumn{2}{|c|}{$\begin{array}{l}\text { Occupational } \\
\text { Change }\end{array}$} & \multirow{2}{*}{$\begin{array}{l}\text { Unadjusted } \\
\text { Demand }\end{array}$} & \multirow[t]{2}{*}{$\begin{array}{c}\text { Percent of } \\
\text { Unadjusted } \\
\text { Demand } \\
\text { Provided } \\
\text { by Net } \\
\text { Adjustment }\end{array}$} \\
\hline & & In & Out & In & Out & & \\
\hline Dentists & 206 & 150 & 0 & 225 & 168 & 1,548 & 13.3 \\
\hline Optometrists & 190 & 35 & 17 & 173 & 0 & 237 & 80.2 \\
\hline Pharmacists & 99 & 99 & 99 & 345 & 247 & 1,808 & 5.5 \\
\hline Physicians, Medical \& Osteopathic & 1,466 & 1,599 & 533 & 895 & 495 & 5,474 & 26.8 \\
\hline Podiatrists & 49 & 33 & 0 & 33 & 16 & 72 & 68.1 \\
\hline Veterinarians & 112 & 56 & 0 & 112 & 56 & 965 & 11.6 \\
\hline Other Health Practitioners & -23 & 0 & 0 & 0 & 23 & 21 & $\overline{0}$ \\
\hline Dietitians & 145 & 61 & 30 & 267 & 152 & 580 & 25.0 \\
\hline Registered Nurses & 199 & 576 & 247 & 683 & 813 & 5,293 & 3.8 \\
\hline Therapists & 233 & 156 & 67 & 311 & 167 & 2,031 & 11.5 \\
\hline Clinical Laboratory Technologists & 239 & 193 & 69 & 308 & 193 & 2,465 & 9.7 \\
\hline Dental Hygienists & 22 & 22 & 5 & 27 & 22 & 258 & 8.5 \\
\hline Health Record Technologists & 41 & 14 & 7 & 48 & 14 & 292 & 14.0 \\
\hline Radiologic Technologists & 7 & 8 & 7 & 23 & 17 & 132 & 5.3 \\
\hline Therapy Assistants & 0 & 0 & 0 & 4 & 4 & 42 & 0.0 \\
\hline Other Health Technologists & 139 & 98 & 21 & 232 & 170 & 827 & 16.8 \\
\hline Clergymen & 566 & 1,045 & 624 & 1,553 & 1,407 & 3,432 & 16.5 \\
\hline Other Religious Workers & 52 & 125 & 73 & 177 & 177 & 499 & 10.4 \\
\hline Economists & 620 & 413 & 59 & 561 & 295 & 1,183 & 52.4 \\
\hline Political Scientists & 0 & 0 & 0 & 16 & 16 & 41 & 0.0 \\
\hline Psychologists & 243 & 262 & 37 & 150 & 131 & 756 & 32.1 \\
\hline Sociologists & 0 & 17 & 0 & 0 & 17 & 39 & 0.0 \\
\hline
\end{tabular}

*Bachelor's degree or higher

Note: Figures in the body of the table may not add precisely to the total net adjustment due to computer rounding of numbers. 
Estimated Average Annual Migration and Occupational Mobility for Workers with College Degrees, Selected Occupations for the Southern Region 1965-1970*

\begin{tabular}{|c|c|c|c|c|c|c|c|}
\hline \multirow[b]{2}{*}{ Occupation } & \multirow{2}{*}{$\begin{array}{c}\text { Total Net } \\
\text { Adjustment }\end{array}$} & \multicolumn{2}{|c|}{ Migration } & \multicolumn{2}{|c|}{$\begin{array}{l}\text { Occupational } \\
\text { Change }\end{array}$} & \multirow{2}{*}{$\begin{array}{l}\text { Unadjusted } \\
\text { Demand }\end{array}$} & \multirow[t]{2}{*}{$\begin{array}{c}\text { Percent of } \\
\text { Unadjusted } \\
\text { Demand } \\
\text { Provided } \\
\text { by Net } \\
\text { Adjustment }\end{array}$} \\
\hline & & In & Out & In & Out & & \\
\hline Urban and Regional Planners & 46 & 31 & 15 & 61 & 31 & 299 & 15.4 \\
\hline Other Social Scientists & 42 & 57 & 42 & 28 & 0 & 230 & 18.3 \\
\hline Social Workers & 1,262 & 402 & 153 & 1,776 & 763 & 4,879 & 25.9 \\
\hline Recreation Workers & 36 & 42 & 18 & 137 & 125 & 339 & 10.6 \\
\hline Agriculture Teachers & 95 & 19 & 0 & 95 & 19 & 68 & 139.7 \\
\hline \multirow{2}{*}{\multicolumn{8}{|c|}{$\begin{array}{l}\text { Atmospheric, Earth, } \\
\text { and Marine Teachers }\end{array}$}} \\
\hline & 53 & 35 & 0 & 35 & 18 & 85 & 62.4 \\
\hline Biology Teachers & 364 & 172 & 57 & 306 & 57 & 553 & 65.8 \\
\hline Chemistry Teachers & 211 & 96 & 58 & 230 & 58 & 174 & 121.3 \\
\hline Physics Teachers & 161 & 71 & 18 & 143 & 36 & 60 & 268.3 \\
\hline Engineering Teachers & 213 & 160 & 35 & 142 & 53 & 175 & 121.7 \\
\hline Mathematics Teachers & 512 & 220 & 55 & 384 & 37 & 559 & 91.6 \\
\hline Health Specialties Teachers & 522 & 87 & 0 & 470 & 35 & 1,034 & 50.5 \\
\hline Psychology Teachers & 176 & 98 & 20 & 137 & 39 & 370 & 47.6 \\
\hline Business and Commerce Teachers & 246 & 38 & 38 & 322 & 76 & 446 & 55.2 \\
\hline Economics Teachers & 176 & 59 & 0 & 118 & 0 & 184 & 95.7 \\
\hline History Teachers & 232 & 77 & 19 & 174 & 0 & 186 & 124.7 \\
\hline Sociology Teachers & 191 & 76 & 19 & 153 & 19 & 274 & 69.7 \\
\hline Other Social Science Teachers & 95 & 57 & 19 & 95 & 38 & 307 & 30.9 \\
\hline Art, Drama, and Music Teachers & 580 & 281 & 53 & 422 & 70 & 683 & 84.9 \\
\hline \multicolumn{8}{|l|}{ Coaches and } \\
\hline Physical Education Teachers & 259 & 104 & 17 & 293 & 121 & 237 & 109.3 \\
\hline
\end{tabular}


TABLE 3-Continued

Estimated Average Annual Migration and Occupational Mobility for Workers with College Degrees, Selected Occupations for the Southern Region 1965-1970*

\begin{tabular}{|c|c|c|c|c|c|c|c|}
\hline \multirow[b]{2}{*}{ Occupation } & \multirow{2}{*}{$\begin{array}{c}\text { Total Net } \\
\text { Adjustment }\end{array}$} & \multicolumn{2}{|c|}{ Migration } & \multicolumn{2}{|c|}{$\begin{array}{l}\text { Occupational } \\
\text { Change }\end{array}$} & \multirow{2}{*}{$\begin{array}{l}\text { Unadjusted } \\
\text { Demand }\end{array}$} & \multirow[t]{2}{*}{$\begin{array}{c}\text { Percent of } \\
\text { Unadjusted } \\
\text { Demand } \\
\text { Provided } \\
\text { by Net } \\
\text { Adjustment }\end{array}$} \\
\hline & & In & Out & In & Out & & \\
\hline Education Teachers & 211 & 115 & 0 & 153 & 57 & 127 & 166.1 \\
\hline English Teachers & 641 & 189 & 94 & 622 & 75 & 688 & 93.2 \\
\hline Foreign Language Teachers & 376 & 215 & 90 & 287 & 36 & 525 & 71.6 \\
\hline Home Economics Teachers & 105 & 35 & 0 & 70 & 0 & 147 & 71.4 \\
\hline Law Teachers & 39 & 0 & 0 & 39 & 0 & 95 & 41.1 \\
\hline Theology Teachers & 75 & 19 & 0 & 75 & 19 & 128 & 58.6 \\
\hline Trade, Indust., \& Technical Teachers & 46 & 23 & 0 & 23 & 0 & 72 & 63.9 \\
\hline Misc. Teachers, College \& Univ. & 334 & 148 & 37 & 260 & 37 & 491 & 68.0 \\
\hline Teachers, Coll. Univ., Subj. Not Spec. & -494 & 1,020 & 510 & 1,497 & 2,501 & 3,290 & - \\
\hline Adult Education Teachers & 500 & 265 & 41 & 531 & 255 & 1,030 & 48.5 \\
\hline Elementary School Teachers & $-8,011$ & 4,924 & 2,409 & 6,619 & 17,145 & 34,350 & - \\
\hline Prekindergarten \& Kinder. Teachers & 1,034 & 388 & 86 & 1,148 & 416 & 4,628 & 22.3 \\
\hline Secondary School Teachers & 10,831 & 2,839 & 733 & 13,502 & 4,776 & 14,110 & 76.8 \\
\hline Other Teachers, Except Coll. \& Univ. & 328 & 146 & 64 & 504 & 258 & 1,102 & 29.8 \\
\hline Agric. and Biological Technicians & 18 & 15 & 3 & 67 & 61 & 117 & 15.4 \\
\hline Chemical Technicians & 101 & 72 & 25 & 249 & 194 & 227 & 44.5 \\
\hline Draftsmen & 64 & 77 & 41 & 241 & 214 & 308 & 20.8 \\
\hline \multicolumn{8}{|l|}{ Elec. and Electronic } \\
\hline Engin. Teachers & 78 & 53 & 17 & 101 & 59 & 243 & 32.1 \\
\hline Industrial Engin. Technicians & -4 & 4 & 2 & 26 & 31 & 11 & - \\
\hline Mechanical Engin. Technicians & -14 & 4 & $1 \overline{4}$ & 11 & 14 & 27 & - \\
\hline Mathematical Technicians & -6 & 0 & 0 & 0 & 6 & 6 & - \\
\hline
\end{tabular}

*Bachelor's degree or higher

Note: Figures in the body of the table may not add precisely to the total net adjustment due to computer rounding of numbers. 
TABLE 3-Continued

Estimated Average Annual Migration and Occupational Mobility for Workers with College Degrees, Selected Occupations for the Southern Region 1965-1970*

\begin{tabular}{|c|c|c|c|c|c|c|c|}
\hline \multirow[b]{2}{*}{ Occupation } & \multirow{2}{*}{$\begin{array}{c}\text { Total Net } \\
\text { Adjustment }\end{array}$} & \multicolumn{2}{|c|}{ Migration } & \multicolumn{2}{|c|}{$\begin{array}{c}\text { Occupational } \\
\text { Change }\end{array}$} & \multirow{2}{*}{$\begin{array}{l}\text { Unadjusted } \\
\text { Demand }\end{array}$} & \multirow[t]{2}{*}{$\begin{array}{c}\text { Percent of } \\
\text { Unadjusted } \\
\text { Demand } \\
\text { Provided } \\
\text { by Net } \\
\text { Adjustment }\end{array}$} \\
\hline & & In & Out & In & Out & & \\
\hline Surveyors & -9 & 20 & 11 & 83 & 101 & 90 & - \\
\hline \multicolumn{8}{|l|}{ Other Engin. \& } \\
\hline Science Technicians & 83 & 64 & 32 & 345 & 294 & 679 & 12.2 \\
\hline Airline Pilots & 223 & 149 & 68 & 223 & 81 & 486 & 45.9 \\
\hline Air Traffic Controllers & 22 & 15 & 4 & 22 & 11 & 37 & 59.5 \\
\hline Embalmers & -9 & 0 & 5 & 33 & 37 & 15 & - \\
\hline Flight Engineers & -13 & 13 & 13 & 7 & 20 & 60 & - \\
\hline Radio Operators & 14 & 2 & 0 & 21 & 9 & 16 & 87.5 \\
\hline \multicolumn{8}{|l|}{ Tool Programmers, } \\
\hline Numerical Control & -11 & 0 & 0 & 6 & 17 & 15 & 一 \\
\hline Other Technicians & -4 & 14 & 8 & 68 & 77 & 165 & - \\
\hline \multicolumn{8}{|l|}{ Vocational and } \\
\hline Educational Counselors & 1,511 & 386 & 80 & 1,624 & 418 & 3,138 & 48.2 \\
\hline Actors & 6 & 12 & 12 & 31 & 25 & 29 & 20.7 \\
\hline Athletes and Kindred Workers & 21 & 47 & 21 & 131 & 135 & 401 & 5.2 \\
\hline Authors & 43 & 43 & 22 & 151 & 129 & 252 & 17.1 \\
\hline Dancers & -3 & 2 & 2 & 3 & 7 & 12 & - \\
\hline Designers & 108 & 133 & 63 & 298 & 260 & 514 & 21.0 \\
\hline Editors and Reporters & 384 & 427 & 110 & 592 & 526 & 1,505 & 25.5 \\
\hline Musicians and Composers & -7 & 37 & 37 & 128 & 135 & 12 & - \\
\hline Painters and Sculptors & 109 & 45 & 35 & 219 & 119 & 354 & 30.8 \\
\hline Photographers & 44 & 23 & 5 & 62 & 37 & 98 & 44.9 \\
\hline
\end{tabular}

*Bachelor's degree or higher

Note: Figures in the body of the table may not add precisely to the total net adjustment due to computer rounding of numbers. 
TABLE 3-Continued

Estimated Average Annual Migration and Occupational Mobility

for Workers with College Degrees, Selected Occupations

for the Southern Region 1965-1970*

\begin{tabular}{|c|c|c|c|c|c|c|c|}
\hline Occupation & $\begin{array}{c}\text { Total Net } \\
\text { Adjustment }\end{array}$ & \multicolumn{2}{|c|}{ Migration } & \multicolumn{2}{|c|}{$\begin{array}{l}\text { Occupational } \\
\text { Change }\end{array}$} & $\begin{array}{l}\text { Unadjusted } \\
\text { Demand }\end{array}$ & $\begin{array}{r}\text { Percent o } \\
\text { Unadjuste } \\
\text { Demand } \\
\text { Provided } \\
\text { by Net } \\
\text { Adjustmen }\end{array}$ \\
\hline Public Rel. Men \& & & & & & & & \\
\hline Publicity Writers & 456 & 143 & 63 & 617 & 242 & 733 & 62.2 \\
\hline Radio and Television Announcers & -27 & 27 & 15 & 23 & 61 & 57 & - \\
\hline Other Writers, Artists, \& Entertainers & 177 & 122 & 67 & 330 & 208 & 532 & 33.3 \\
\hline $\begin{array}{l}\text { Research Workers, } \\
\text { Subj. Not Specified }\end{array}$ & -257 & 614 & 414 & 599 & 1,056 & 2,360 & - \\
\hline Managers and Administrators & 19,813 & 6,810 & 3,126 & 38,810 & 22,682 & 83,891 & 23.6 \\
\hline $\begin{array}{l}\text { Misc. Public Officials \& Admin. } \\
\text { Sales Managers, Nonretail Trade } \\
\text { School Admin., Elem. \& Secondary } \\
\text { Others Managers and Administrators }\end{array}$ & $\begin{array}{r}1,441 \\
1,684 \\
2,279 \\
\quad 14,409\end{array}$ & $\begin{array}{r}532 \\
640 \\
129 \\
5,509\end{array}$ & $\begin{array}{r}170 \\
394 \\
165 \\
2,397\end{array}$ & $\begin{array}{r}2,165 \\
2,609 \\
3,492 \\
30,544\end{array}$ & $\begin{array}{r}1,086 \\
1,172 \\
1,176 \\
19,248\end{array}$ & $\begin{array}{r}2,912 \\
3,712 \\
3,561 \\
73,706\end{array}$ & $\begin{array}{l}49.5 \\
45.4 \\
64.0 \\
19.5\end{array}$ \\
\hline Sales & 2,696 & 2,389 & 995 & 20,652 & 19,349 & 18,908 & 14.3 \\
\hline $\begin{array}{l}\text { Insurance Agents, } \\
\text { Brokers \& Underwriters } \\
\text { Real Estate Agents and Brokers } \\
\text { Sales Representatives, Manufacturing } \\
\text { Sales Representatives, Wholesale } \\
\text { Other Sales }\end{array}$ & $\begin{array}{l}663 \\
531 \\
338 \\
275 \\
889\end{array}$ & $\begin{array}{l}397 \\
185 \\
550 \\
421 \\
836\end{array}$ & $\begin{array}{r}146 \\
32 \\
263 \\
203 \\
351\end{array}$ & $\begin{array}{r}2,119 \\
882 \\
4,745 \\
5,121 \\
7,785\end{array}$ & $\begin{array}{r}1,707 \\
503 \\
4,695 \\
5,064 \\
7,380\end{array}$ & $\begin{array}{l}2,242 \\
2,352 \\
2,529 \\
2,484 \\
9,301\end{array}$ & $\begin{array}{r}29.6 \\
22.6 \\
13.4 \\
11.1 \\
9.6\end{array}$ \\
\hline Clerical & 3,283 & 1,853 & 757 & 9,575 & 7,389 & 22,233 & 24.5 \\
\hline
\end{tabular}

*Bachelor's degree or higher

Note: Figures in the body of the table may not add precisely to the total net adjustment due to computer rounding of numbers. 
TABLE 3-Continued

Estimated Average Annual Migration and Occupational Mobility

for Workers with College Degrees, Selected Occupations

for the Southern Region 1965-1970*

\begin{tabular}{|c|c|c|c|c|c|c|c|}
\hline \multirow{2}{*}{ Occupation } & \multirow{2}{*}{$\begin{array}{c}\text { Total Net } \\
\text { Adjustment }\end{array}$} & \multicolumn{2}{|c|}{ Migration } & \multicolumn{2}{|c|}{$\begin{array}{l}\text { Occupational } \\
\text { Change }\end{array}$} & \multirow{2}{*}{$\begin{array}{l}\text { Unadjusted } \\
\text { Demand }\end{array}$} & \multirow[t]{2}{*}{$\begin{array}{c}\text { Percent of } \\
\text { Unadjusted } \\
\text { Demand } \\
\text { Provided } \\
\text { by Net } \\
\text { Adjustment }\end{array}$} \\
\hline & & In & Out & In & Out & & \\
\hline Bookkeepers & 878 & 205 & 74 & 1,455 & 708 & 3,215 & 27.3 \\
\hline Secretaries & 786 & 416 & 158 & 1,250 & 723 & 4,345 & 18.1 \\
\hline Misc. and Not Specified Clerical & 1,619 & 1,232 & 525 & 6,870 & 5,958 & 14,673 & 25.8 \\
\hline Other Occupations & 912 & 1,275 & 825 & 10,877 & 10,412 & 17,420 & 5.2 \\
\hline Miscellaneous Foremen & 1,662 & 227 & 93 & 2,747 & 1,219 & 2,433 & 68.3 \\
\hline Farm Owners and Tenants & -540 & 34 & 53 & 486 & 1,008 & 1,437 & - \\
\hline Other & -210 & 1,014 & 679 & 7,644 & 8,185 & 13,550 & - \\
\hline
\end{tabular}

*Bachelor's degree or higher

Note: Figures in the body of the table may not add precisely to the total net adjustment due to computer rounding of numbers. 
Under the assumption that the 1965-70 experience will extend into the 1970's this level of movement would fulfill 20 percent of the average annual demand for college educated workers projected for the 1970-80 decade. This finding alone suggests that occupational and geographic mobility are serious supply components to be dealt with in regional higher education manpower supply and demand studies. This conclusion is strengthened in the findings for individual occupations. Table 4 summarizes the extent to which projected demand in the 138 predominantly professional and managerial occupations may be fulfilled by migration and occupational change. At least half of the demand would be satisfied by the two types of mobility in 43 occupations and the net additional supply from mobility could consume 0-50 percent of the annual demand in 73 other occupations. Only in 22 occupations would the adjustment for job and geographic mobility enhance the demand picture rather than detract from it, with many of these occupations falling in the general category of skilled technicians.

\section{TABLE 4}

Summary Tally of Occupations by Percentage of Demand Fulfilled by Net Adjustment

\begin{tabular}{cc}
\hline Percentage of & Number of \\
Demand Fulfilled & Occupations \\
\hline Total & 138 \\
100 and over & 11 \\
$80-99$ & 6 \\
$50-79$ & 26 \\
$0-50$ & 73 \\
Negative & 22 \\
\hline
\end{tabular}

A second general pattern that may be observed in Table 3 is the tendency for occupational change to account for slightly more of the total net adjustment than does migration. There is substantial variation among occupations, with the range extending from well over 100 percent (i.e., occupational change more than overcomes the migration adjustment in the opposite direction) to the reverse situation in which the adjustment is totally dictated by the migration factor. In large part the occupational change adjustment results in an additional net supply (that is the number of in-tranfers is greater than out-transfers). In 28 occupations, however, out-transfers exceed in-tranfers, creating potentially open positions rather than fostering additional supplies of workers. The most noteworthy cases, due to the volume of workers involved, are registered nurses, university instructors of unspecified disciplines, elementary school teachers, general research workers, farm owners and tenants, and the miscellaneous "other" category. It is also a general finding that where a negative total net adjustment is observed, occupational change, rather than migration, is responsible. 
A concentration on net adjustments masks perhaps the most significant trend in Table 3, however; the extreme amount of interoccupational movement, throughout the job spectrum, relative to the levels of gross activity in geographic migration approximately 255,000 in and out shifts of jobs occurred annually; over four times the gross migration of 60,000 persons.

Comparisons of gross levels (ins plus outs) may misrepresent the situation somewhat, in that occupational transfers are double counted. That is, a job transfer by one person is tabulated as both an out-transfer from his previous occupation and an in-transfer to his new position. Nonetheless, even reducing the 255,000 job changes by one-half, the level of activity is still double that of migration. This ratio may shift substantially, however, with the adverse economic conditions of the early 1970's tending to retard occupational movement, and with 1970-75 Southern migration reaching well above historical levels. Nonetheless, it is evident that in the long run interoccupational movement is one of the more dynamic and effective forces in labor market supply-demand interaction.

Finally, it may be noted also from Table 3 that the occupations indicating net outmigration of workers rarely show a similar net outflow of workers from occupational transfers. This lack of correspondence in occupations with similar occupational mad migration loss patterns suggests that the two factors may serve as differing types of market adjustment mechanisms.

\section{Reservations and Limitations}

None of the general findings may be totally dismissed on the grounds of technical difficulty or may be explained away as mere data aberrations. Nonetheless, there are some accompanying limitations that must be considered. First is the inference that the positions vacated by outmigrants and out-transfers of workers will be filled. Within the context of short-term difficult economic circumstances, this practice may not be followed, but it is a comfortable provision in long-run projections extending over a 10-year period. Second, it is also implied in defining worker supplies that workers transferring into jobs and migrants to the Southern region and states have positions waiting for them. There is no difficulty in accepting the transfer provision, since by definition the worker would not be transferring if the open position were not available. While the assumption on in-migrants may not seem entirely justified, this analysis deals only with college graduates with the much greater likelihood that the assumption would hold than for workers in general.

Third, some attention must be given to the basic data source for the two supply adjustment factors. Although the Public Use Sample files were found to be a valuable resource for the adjustment variables, the sampling error in the original census migration and transfer returns is sufficient that it should be taken into account when encountering as 
few cases as are often shown in Table 1. The use of the PUS files for the migration and occupational transfer responses also constitutes a sample of a sample, i.e., a one percent sample of respondents to what was originally a sample question.

A related issue is raised in a Census Bureau working paper concerning the basic adequacy of response and coding of the census occupational movement question (Walsh and Buckholt, 1970). Their conclusion is that the primary shortcoming of the data is an intolerably imprecise recall of occupational activities five years earlier by respondents, particularly for occupationally mobile individuals. Their contention is that retrospective questions such as used in their evaluation study substantially understate mobility, but matching studies (i.e., actual measurement of occupation at two discrete points in time) tend to over-estimate movement. The "true" mobility rate is anticipated to fall somewhere between the two.

There is some comfort in the finding that 1970 PUS rates for the Southern region do, in large part, lie between the two rates developed in the Walsh and Buckholt study of U.S. patterns. Table 5 indicates that the PUS rates generally more closely approximate what would be expected from a retrospective data collection procedure, but it may also be seen that the rates for only three of the twelve occupations (including total) violate the range of rates set by the retrospective and matched results.

Further support may be found in the nature of the results themselves. It must be kept in mind that the focus here is on general magnitude and occupational structure of the net adjustments rather than individual numbers for isolated detailed occupations. Within this objective, the profile of adjustments appears reasonable. For example, an informed observer might expect a large influence from occupational and geographic mobility for elementary and secondary teachers, the business community (managers and administrators, personnel workers), computer fields, some engineering occupations, physicians, and social workers. Large adjustments are found for these occupations in the 1970 PUS data. Similarly, the percentage of adjustment due to occupational mobility fits with common knowledge. For example, job changes are high for managers and administrators and clerical but are lower for the professional category. Among the professional group, job movement is high for computer specialists, nurses, and teachers. In addition the negative (i.e., out-transfers exceed in-transfers) categories fall in occupations where they might be expected. In short, there are too many pieces of supporting evidence to totally dismiss the structure of the findings as error-ridden and capricious.

Finally, the retention of 1965-1970 migration and occupational change levels is appropriate for testing purposes but is not likely to anticipate accurately the events of the 1970-1980 decade. The conditions responsible for such a departure from recent experience and the indications that 


\section{TABLE 5}

Comparison of Occupational Mobility Rates; Matched Data for the U.S., Retrospective Current Population Survey Study for the U.S., and 1970 Census Public Use Sample Data for the South

\begin{tabular}{lccc}
\multicolumn{3}{c}{ Occupational Mobility Rates* } \\
\hline \multicolumn{1}{c}{ Occupation } & $\begin{array}{c}1963-68 \\
\text { Matched } \\
\text { Data }\end{array}$ & $\begin{array}{c}1963-68 \\
\text { Retrospective } \\
\text { Data }\end{array}$ & $\begin{array}{c}\text { 1965-70 Census } \\
\text { PUS Data }\end{array}$ \\
\hline Total & 31.5 & 15.9 & 17.8 \\
Professionals & 16.4 & 6.9 & 10.7 \\
Farmers & 26.0 & 18.3 & 19.9 \\
Managers & 32.8 & 12.9 & 19.1 \\
Clerical Workers & 26.5 & 12.4 & 13.3 \\
Sales Workers & 42.9 & 28.6 & 23.9 \\
Craftsmen & 26.8 & 13.6 & 15.1 \\
Operatives & 28.7 & 16.5 & 20.7 \\
Private Household & 34.8 & 18.2 & 13.8 \\
Service Workers & 35.2 & 20.9 & 17.1 \\
Farm Laborers & 46.6 & 22.0 & 24.0 \\
Nonfarm Laborers & 61.3 & 32.1 & 33.8 \\
\hline
\end{tabular}

*Rates for matched and CPS retrospective data refer to workers age 19 and over from Walsh and Buckholt, 1970. Census PUS rates are for workers age 21 and over.

the break has in fact occurred for the migration component have been discussed at some length earlier. It remains that due to a number of considerations, we may not identify precisely the migration and occupational change that will be experienced but can establish their potential impact within some approximate bounds.

\section{Summary and Conclusions}

The purpose of this analysis has been (1) to gauge the potential importance of occupational mobility and geographic migration as "noise" variables in the comparison of the projected supply and demand for college graduates in the South, and (2) to establish a preliminary expectation as to the occupational profile of the adjustments that might follow.

Even recognizing potential hazards in the underlying assumptions that are necessary, and granting some shortcomings with the nature of the basic occupational movement data, it is clear that these two mobility factors must be contended with as disruptive variables in the seemingly straightforward evaluation of the professionally trained worker supply and demand. The two mobility factors combined constitute a supply component capable of satisfying significant amounts of the demand projected for most occupations. An extreme amount of interjob movement was found, far overshadowing the gross levels of in and out migration. Although increased attention must be given to migration due to the escalated migratory activity during the early 1970's, the analysis verifies that occupational mobility may not be ignored as a major factor to be dealt with in Southern supply and demand studies. 
The findings also identified a differential importance of the two mobility factors for many occupations, suggesting varying roles for them in the supply-demand market adjustment process, and pinpointing the occupations needing special attention in adjusting worker supplies.

\section{REFERENCES}

Alabama Commission on Higher Education, Alabama College-Level Manpower: A Preliminary Study of Supply and Demand (Mobile, Alabama: State of Alabama, 1973), pp. 44-47.

Engels, Richard A., "Higher Education SupplyDemand Projections for States: Evaluation of Some Proposed Features and Data," The Review of Public Data Use, Vol. 3, Number 4, October 1975.

Lee, Joe Won and William B. D. Hong, Regional Economic Projections: 1960-85 (Washington, D.C.: The National Planning Association, Report No. 73-5-1, December 1973).

Long, John F. and Celia G. Boertlein (U.S. Bureau of the Census), "Trends in Migration by Occupation," unpublished paper presented at the annual meeting, Southern Regional Demographic Group, Atlanta, Georgia, October 1975.

Rake, Charles Penley, Supply of and Demand for New Labor Force Entrants in Colorado: Regional Projection Analysis, 1970-1980 (Boulder, Colorado: Business Research Division, University of Colorado, undated), pp. 1, 6-7, and 97-99.

Siler, Pamela, "Manpower/Education Correlation System," (Salem, Oregon: Oregon Edu- cational Coordinating Council, undated), pp. 36-37.

U.S. Bureau of the Census, Public Use Samples of Basic Records from the 1970 Census: Description and Technical Documentation (Washington, D.C.: Social and Economic Statistics Administration, 1972).

U.S. Bureau of Labor Statistics, Tomorrow's Manpower Needs, Volume 1 (U.S. Government Printing Office, Washington, D.C. 20402) p. 50.

U.S. Bureau of Labor Statistics, Occupational Supply: Concepts and Sources of Data for Manpower Analysis (Bulletin 1816), (Washington, D.C.: U.S. Government Printing Office, 1974), pp. 22-24.

Walsh, Thomas C. and Paula J. Buckholt (U.S. Bureau of the Census), "Accuracy of Retrospectively Reporting Work Status and Occupation Five Years Ago," unpublished working paper number E15 No. 3, February 19, 1970.

White, Fred C., and H. Evan Drummond, Manpower Requirements for Georgia, 1970-1980," unpublished paper available through the Department of Agricultural Economics, the University of Georgia, pp. 6-8. 